\title{
Association between retinal neuronal degeneration and visual function impairment in type 2 diabetic patients without diabetic retinopathy
}

\author{
ZHU TiePei ${ }^{1}$, MA Jin ${ }^{2 *}$, LI YongHao ${ }^{2} \&$ ZHANG Zheng ${ }^{3}$ \\ ${ }^{1}$ Eye Hospital of Wenzhou Medical University, Hangzhou 310020, China; \\ ${ }^{2}$ State Key Laboratory of Ophthalmology, Zhongshan Ophthalmic Center, Sun Yat-sen University, Guangzhou 510060, China; \\ ${ }^{3}$ Eye Center, Second Affiliated Hospital of Medical College, Zhejiang University, Hangzhou 310008, China
}

Received December 11, 2014; accepted March 8, 2015; published online May 6, 2015

\begin{abstract}
The changes in retinal thickness and visual function in type 2 diabetic patients without clinical evidence of diabetic retinopathy were evaluated. A total of 141 diabetic subjects without retinopathy and 158 healthy subjects were enrolled in this study. Superior macular ganglion cell complex thicknesses were significantly decreased in diabetic cases, and no significant peripapillary retinal nerve fiber layer thickness changes were observed. The contrast sensitivities at all space frequencies were significantly different between diabetic patients and controls. The mean P50 amplitude from pattern electroretinogram results was reduced significantly in the diabetic group. In the diabetic group, average superior ganglion cell complex thicknesses positively correlated with both contrast sensitivities at high spatial frequencies and P50 amplitudes. The results indicated that ganglion cell complex thickness and visual function changes could be observed in diabetic subjects before the onset of any significant diabetic retinopathy. Macular ganglion cell complex reduction occurred much earlier than peripapillary retinal nerve fiber layer thinning in diabetic patients without retinopathy.
\end{abstract}

diabetes mellitus, diabetic retinopathy, retinal nerve fiber layer, retinal ganglion cell, contrast sensitivity, electroretinogram

Citation: Zhu TP, Ma J, Li YH, Zhang Z. Association between retinal neuronal degeneration and visual function impairment in type 2 diabetic patients without diabetic retinopathy. Sci China Life Sci, 2015, 58: 550-555, doi: 10.1007/s11427-015-4858-8

Diabetic retinopathy (DR) is one of the major complications in patients with diabetes mellitus (DM) that can lead to blindness. DR has been considered primarily a retinal microvascular disorder caused by the direct effects of hyperglycemia and by the metabolic pathways it activates [1]. Previous studies have shown that vascular abnormalities and neuronal alterations, including neural apoptosis, loss of ganglion cells, glial reactivity, and reduction in thickness of the inner retinal layers, accompany the pathogenic changes at the earliest stages of DR [2-5]. Moreover, structural

*Corresponding author (email: zoc_majin@aliyun.com) neuropathy can occur prior to the onset of visible diabetic vasculopathy, as shown in earlier experimental studies $[5,6]$. Structural alterations of retinal nerve tissue have also been reported in several clinical studies, as evidenced by thinning of the retinal nerve fiber layer (RNFL) in diabetic patients without diabetic retinopathy (NDR) compared with nondiabetic patients [7-9].

In addition to typical retinopathies, neurovisual impairments have been reported. Abnormalities in visual evoked potentials, contrast sensitivity, and dark adaption and electroretinogram (ERG) scans have been assessed in diabetic patients and indicated neuronal system involvement 
[2,10-14]. Morphological changes of retinal nerve tissue may explain the neurovisual functional deficits that are observed in diabetic patients, even before the overt retinopathy is seen. However, to the best of our knowledge, relationships between early retinal structure alterations and visual function impairments in a same population of NDR have not been reported. The purpose of our study was to evaluate the hypothesis that RNFL thickness and ganglion cell complex (GCC) thickness measured by spectral domain optical coherence tomography (SD OCT) and the visual functions tested with contrast sensitivity and pattern ERG (PERG) were associated in NDR patients.

\section{Materials and methods}

\subsection{Study participants}

This was a cross-sectional, case-control study. Patients were randomly recruited from the outpatient clinic of the Zhongshan Ophthalmic Center (Sun Yat-Sen University, Guangzhou). Inclusion criteria were type $2 \mathrm{DM}$ patients without any signs of DR as evaluated by a retinal specialist ( $\mathrm{J} \mathrm{Ma)} \mathrm{through} \mathrm{indirect} \mathrm{fundoscopy} \mathrm{and} \mathrm{stereo} \mathrm{color} \mathrm{fundus}$ photography, according to the International Clinical Diabetic Retinopathy Disease Severity Scale [15]. Exclusion criteria were clinically observed DR, myopia more than six diopters, visual acuity below 0.1 , intraocular pressure (IOP) higher than $21 \mathrm{mmHg}$, history of glaucoma, uveitis, or retinal disease, or previous history of laser or intraocular surgery. Age- and sex-matched subjects, free of ocular disease, diabetes, hypertension, or other systemic diseases were recruited as controls from among those who accompanied patients visiting the outpatient clinic. The study was approved by the Zhongshan Ophthalmic Center institutional review board and conformed to the tenets of the Declaration of Helsinki, and all participants gave written informed consent.

Each subject underwent a complete ophthalmic examination, including determining best-corrected visual acuity (BCVA), refraction measurement, IOP measurement by noncontact tonometry, slit-lamp biomicroscopy, and dilated fundus examinations. All subjects were divided into two study groups, the NDR and control group, and NDR was defined as the absence of all features of DR [15].

\subsection{SD OCT measurements}

OCT was performed to obtain measurements of the peripapillary RNFL thickness using the RTVue-100 device (Optovue, Inc., Fremont, CA, USA). One eye of each subject was randomly chosen for measurement, and every eye was repeatedly measured three times. The peripapillary thickness was determined by optical nerve head mode, in which data along a 3.4-mm diameter circle around the optic disc were recalculated with a map created from en face im- aging, using six circular and 12 linear data inputs. Average, superior, and inferior RNFL thicknesses were calculated. The GCC parameters were obtained by the macular map protocol, centered $1 \mathrm{~mm}$ temporal to the fovea. This protocol used one horizontal line with a 7-mm scan length (934 A-scans), followed by 15 vertical lines with a 7-mm scan length and 0.5-mm interval (800 A-scans). The GCC thickness was measured from the internal limiting membrane to the inner plexiform layer boundary; average, superior, and inferior GCC thicknesses were calculated.

\subsection{Contrast sensitivity test}

Contrast sensitivity was evaluated with the OPTC 6500 (Stereo Optical; Chicago, IL, USA). This test provided presentation of sine-wave gratings of different spatial frequencies $(1.5,3,6,12$, and 18 cycles per degree (cpd)) with a contrast-level change step corresponding to $0.15 \mathrm{log}$ contrast sensitivity. Following the manufacturer's protocol, the testing distance was $3 \mathrm{~m}$ for distance. The optimum additional spectacle corrections were used and an evaluation of the contrast sensitivity test was done monocularly in each subject. The contrast sensitivity measurements were performed under a constant luminance condition of $85.0 \mathrm{~cd} \mathrm{~m}^{-2}$.

\subsection{PERG}

PERG was performed according to the International Society for Clinical Electrophysiology of Vision standard for clinical patterns [16]. In brief, gold foil corneal recording electrodes were positioned directly under the center of the pupil so that there was no movement of the electrode when the patient blinked. Reference and ground electrodes were placed in the outer canthus and on the forehead, respectively. A black-and-white reverse checkerboard pattern was used with an aspect ratio of width over height of the stimulus field not exceeding 4:3. The mean of the width and height of the stimulus field was 15 degrees, with a check size of 0.8 degrees. The contrast between black and white squares was close to $100 \%$. The reversal rate was $2.2 \mathrm{~Hz}$. A minimum of 100 artifact-free sweeps was collected and averaged. The P50 amplitude was calculated from the trough of N35 to the peak of P50. All data were recorded without pupil dilation.

\subsection{Data analyses}

Descriptive statistical data were described as the mean with standard deviation. All statistical analyses were performed using SPSS software version 17.0 (SPSS Co., Chicago, IL, USA). The distributions of characteristic data such as age, sex, and IOP in two groups were checked by using $\chi^{2}$ tests or unpaired $t$-tests, as appropriate. For the statistical comparison of RNFL thickness and GCC thickness between two 
groups, the ANOVA analysis was used. Correlation analyses were performed using Pearson's correlation coefficient for parametric data. $P$ values less than 0.05 were considered as statistically significant.

\section{Results}

\subsection{General characteristics}

A total of 141 eyes from 141 NDR subjects and 158 eyes from 158 healthy subjects met our criteria and were included in this study. The characteristics of the two groups are presented in Table 1. No significant difference was found for age, sex, and IOP among groups.

\subsection{Peripapillary RNFL and macular GCC thickness}

After adjusting for age and gender, the average, superior

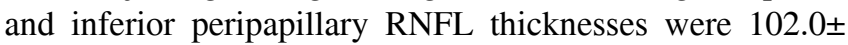
$12.1 \mu \mathrm{m}, 128.5 \pm 15.3 \mu \mathrm{m}$, and $124.1 \pm 14.9 \mu \mathrm{m}$ in controls, respectively, and $99.2 \pm 14.7 \mu \mathrm{m}, 126.0 \pm 16.1 \mu \mathrm{m}$, and $120.0 \pm 15.3 \mu \mathrm{m}$ in NDR subjects, respectively. The average, superior and inferior macular GCC thicknesses were $101.6 \pm 10.2 \mu \mathrm{m}, 111.3 \pm 11.1 \mu \mathrm{m}$, and $110.2 \pm 10.2 \mu \mathrm{m}$ in controls, respectively, and 99.3 $\pm 9.5 \mu \mathrm{m}, 103.2 \pm 13.2 \mu \mathrm{m}$, and $106.0 \pm 11.3 \mu \mathrm{m}$ in NDR subjects, respectively. Differences in all RNFL parameters between NDR and control eyes were not statistically significant. The macular GCC thickness in the NDR eyes was significantly reduced by $6.8 \%$ in the superior region (ANOVA, $P<0.05$ ), whereas no significant difference was found in the inferior area (Figure 1).

\subsection{Visual function}

Table 2 shows visual function parameters in the two groups, including BCVA, contrast sensitivity values, P50 implicit times, and amplitudes from PERG. According to Table 2, all data showed normal distributions upon KolmogorovSmirnov normality testing. There was no significant difference in BCVA among the control and NDR groups. Contrast sensitivity values were significantly decreased at every spatial frequency in the NDR versus control groups

Table 1 Clincal characteristics of normal and NDR subjects ${ }^{\mathrm{a}}$

\begin{tabular}{cccc}
\hline Characteristics & Normal & NDR & $P$ value \\
\hline Case number & 158 & 141 & - \\
Male sex, $n(\%)$ & $86(54.4)$ & $76(53.9)$ & 0.91 \\
Age (year), mean \pm SD & $63.62 \pm 10.57$ & $68.10 \pm 9.33$ & 0.38 \\
IOP (mmHg), mean \pm SD & $16.05 \pm 2.25$ & $14.53 \pm 2.50$ & 0.22 \\
Diabetes duration (year) & - & $9.75 \pm 2.11$ & - \\
\hline
\end{tabular}

a) NDR, no diabetic retinopathy; IOP, intraocular pressure. Age and IOP were analyzed by $t$ test, male sex was analyzed by Chi-square test.

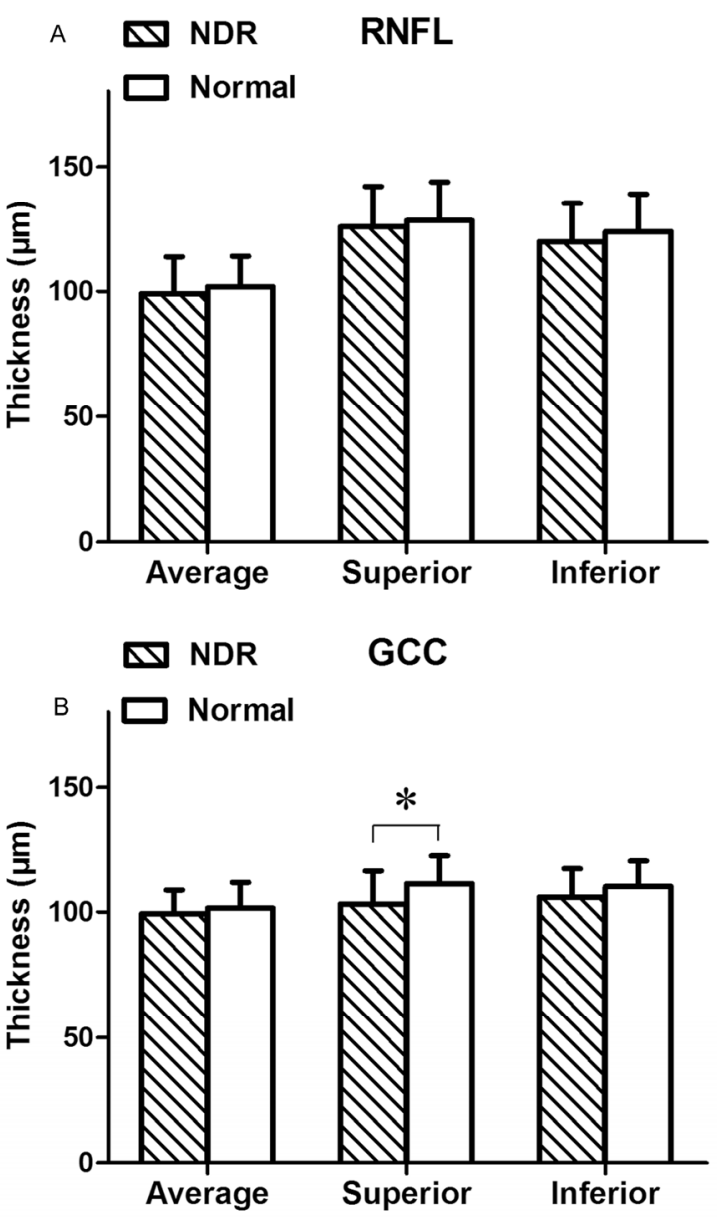

Figure 1 Peripapillary retinal nerve fiber layer (RNFL) thickness (A) and macular ganglion cell complex (GCC) thickness (B) measured by spectral-domain optic coherence tomography in diabetic patients without diabetic retinopathy (NDR) and in normal subjects. The asterisk indicates statistically different changes.

(ANOVA, all $P<0.05$ ). Results of PERG showed that the P50 amplitudes were significantly different between groups (ANOVA, $P<0.05$ ), whereas the P50 implicit time was not changed significantly.

\subsection{Correlations}

In NDR eyes, significant positive correlations were found between superior macular GCC and contrast sensitivity at spatial frequencies of $6,12,18 \mathrm{cpd}(r=0.51,0.52$, and 0.49 , respectively; all $P<0.05)$, and lower positive correlations were also found between inferior RNFL and contrast sensitivity at high spatial frequencies of 12 and $18 \mathrm{cpd}(r=0.3$ and 0.25 , respectively; $P<0.05$ ). For the PERG data, there were positive correlations between P50 amplitudes and both inferior RNFL thickness and superior GCC thickness ( $r=0.65$ and 0.41 , respectively; both $P<0.05$ ). Interestingly, no significant correlation was found between P50 implicit times and any retinal structure parameter (Table 3 ). 
Table 2 Comparison of visual function parameters in normal and NDR subjects ${ }^{\text {a) }}$

\begin{tabular}{|c|c|c|c|c|c|c|c|c|}
\hline & \multirow{2}{*}{$\begin{array}{c}\text { BCVA } \\
\text { (LogMAR) }\end{array}$} & \multicolumn{5}{|c|}{ Contrast sensitivity } & \multicolumn{2}{|c|}{ PERG } \\
\hline & & $1.5 \mathrm{cpd}$ & $3 \mathrm{cpd}$ & $6 \mathrm{cpd}$ & $12 \mathrm{cpd}$ & $18 \mathrm{cpd}$ & Amplitude $(\mu \mathrm{m})$ & Implicit time (ms) \\
\hline NDR & $0.10 \pm 0.19$ & $37.78 \pm 11.06$ & $49.02 \pm 10.81$ & $61.21 \pm 15.09$ & $30.22 \pm 4.73$ & $10.19 \pm 1.26$ & $0.91 \pm 0.16$ & $63.29 \pm 8.15$ \\
\hline Normal & $0.08 \pm 0.11$ & $49.01 \pm 10.77$ & $64.11 \pm 13.08$ & $79.33 \pm 16.21$ & $45.5 \pm 8.70$ & $17.55 \pm 2.62$ & $1.42 \pm 0.20$ & $61.11 \pm 8.39$ \\
\hline$P$ value ${ }^{*}$ & 0.82 & 0.03 & 0.04 & 0.01 & 0.01 & 0.02 & 0.02 & 0.15 \\
\hline
\end{tabular}

a) NDR, no diabetic retinopathy; BCVA, best corrected visual acuity; cpd, cycles per degree; pERG, pattern electroretinogram. Data are reported as mean \pm SD. Bold font indicates significant difference between NDR and normal groups. *, ANOVA analysis.

Table 3 Correlations between retinal neural thicknesses and visual function parameters in NDR subjects ${ }^{\text {a) }}$

\begin{tabular}{|c|c|c|c|c|c|c|c|c|}
\hline \multirow{2}{*}{ Parameters } & & \multicolumn{5}{|c|}{ Contrast sensitivity } & \multicolumn{2}{|c|}{ PERG } \\
\hline & & $1.5 \mathrm{cpd}$ & $3 \mathrm{cpd}$ & $6 \mathrm{cpd}$ & $12 \mathrm{cpd}$ & $18 \mathrm{cpd}$ & Amplitude $(\mu \mathrm{m})$ & Implicit time (ms) \\
\hline Average RNFL & $r$ & 0.19 & 0.13 & 0.11 & 0.16 & 0.34 & 0.11 & 0.29 \\
\hline \multirow[t]{2}{*}{ Superior RNFL } & $r$ & 0.16 & 0.07 & 0.13 & 0.14 & 0.17 & 0.19 & 0.22 \\
\hline & $P$ value & 0.15 & 0.51 & 0.32 & 0.22 & 0.17 & 0.09 & 0.09 \\
\hline Inferior RNFL & $r$ & 0.12 & 0.19 & 0.22 & 0.3 & 0.25 & 0.65 & 0.19 \\
\hline \multirow[t]{2}{*}{ Average GCC } & $r$ & 0.13 & 0.14 & 0.19 & 0.22 & 0.17 & 0.16 & 0.07 \\
\hline & $P$ value & 0.32 & 0.22 & 0.09 & 0.09 & 0.17 & 0.15 & 0.51 \\
\hline \multirow[t]{2}{*}{ Superior GCC } & $r$ & 0.2 & 0.24 & 0.51 & 0.52 & 0.49 & 0.41 & 0.32 \\
\hline & $P$ value & 0.13 & 0.11 & 0.01 & 0.01 & 0.01 & 0.04 & 0.07 \\
\hline \multirow[t]{2}{*}{ Inferior GCC } & $r$ & 0.12 & 0.13 & 0.24 & 0.19 & 0.32 & 0.19 & 0.2 \\
\hline & $P$ value & 0.33 & 0.32 & 0.11 & 0.13 & 0.07 & 0.09 & 0.11 \\
\hline
\end{tabular}

a) NDR, no diabetic retinopathy; BCVA, best corrected visual acuity; cpd, cycles per degree; pERG, pattern electroretinogram. Data are reported as mean \pm SD. Bold font indicates significant correlation.

\section{Discussion}

In this study we demonstrated that macular GCC thickness was significantly correlated with visual function parameters, such as contrast sensitivity and PERG amplitudes, in NDR patients compared with control subjects. To our knowledge, this is the first study exploring the structure-function relationships of the retina in diabetic patients before the onset of DR.

Previous work suggested that early neuronal degeneration in the retina could occur even though the retinal vascular lesions in diabetic patients were minimal. The inner retina, which is supplied by the retinal vessels, seems to be more vulnerable to metabolic stress induced by diabetes, compared with the outer retina, which is supplied by the choroidal circulatory system [17]. Therefore, in our study the peripapillary RNFL and macular GCC thicknesses were measured by SD OCT to detect early neuronal degeneration. It is well known that RNFL and GCC thicknesses decreased with age $[18,19]$. As shown in Table 1 , there was no significant difference with respect to age between diabetic and normal subjects. Moreover, to exclude the possibility that retinal inner layer thickness was decreased with age, in this study we adjusted the ages within the study groups.

In the present study, we did not find significant differences in peripapillary RNFL thicknesses between the NDR and normal groups, although there was a trend of sectional
RNFL reduction in NDR patients versus controls. This finding was consistent with other studies of preclinical DR [20-22]. One possible reason may be the high density of retinal nerve fibers in the peripapillary region [22]. However, significant thinning of the peripapillary RNFL thickness in preclinical DR has been reported in some previous studies $[8,23,24]$. This discrepancy between studies may be due to the difference in patient populations, devices for RNFL measurements, or study designs. It has been suggested that the peripapillary RNFL thickness profiles measured with OCT vary among individuals, and this variation was large relative to other factors, such as measurement error [25,26], optic disc size [27], or the retinal arterioles and venules extending from the optic disc [28].

In addition to peripapillary RNFL, we also evaluated macular GCC thickness to detect inner retinal damage. The macular region may be more susceptible to diabetic damage, because of its higher metabolic demands when compared with the peripapillary region [22]. The GCC thickness measured in the present study consisted of the macular RNFL, ganglion cell layer and inner plexiform layer. As reported by Kim et al. [29], the GCC thickness was a more direct measure of the integrity of retinal ganglion cells, and could be considered a better indicator of early retinal neuronal damage than peripapillary RNFL. Although the peripapillary RNFL thickness was not changed in the NDR group, in this study we observed a significant decrease of up 
to $6.8 \%$ in superior macular GCC thickness in NDR patients compared with controls. Similar results have been reported previously [8,30,31]. Our finding of macular GCC loss could be explained by the loss of retinal ganglion cells and axons prior to the thinning of the RNFL $[32,33]$. In addition, the superior region of the macular is more susceptible to initial diabetic damage than the other regions. A previous diabetic animal model study reported that the superior area had twice the number of microaneurysms and cellular capillaries compared with the inferior area in the retina [34], suggesting a different retinopathy in the superior versus inferior regions. However, further studies are needed to determine the cause of the reduction in macular GCC thickness in diabetic patients without clinical DR.

The BCVA did not differ between NDR and normal groups in the present study. We hypothesize that the slight macular GCC loss in NDR patients may be insufficient to detect significant BCVA changes. Previous contrast sensitivity tests revealed abnormal functions before visual acuity tests, and correlated positively with the presence and the degree of diabetic retinopathy [35]. Several contrast sensitivity studies in diabetic patients showed a general decrease of sensitivity across low, medium, and high spatial frequencies, suggesting that both the parvocellular and magnocellular systems involved in contrast processing were affected by the disease [12,35-37]. Similar results were found in our study, where contrast sensitivities at each spatial frequency (from 1.5 to $18 \mathrm{cpd}$ ) were significantly decreased in NDR patients, compared with normal subjects. It has been suggested that the contrast abnormality occurring before the onset of the DR may be due to early retinal neural degeneration [37-39]. Interestingly, in our study we found that only contrast sensitivities at high spatial frequencies were positively correlated with macular GCC and peripapillary RNFL thickness. These correlations are expected because previous studies indicated that the selective losses at high spatial frequencies may be due to the dysfunction in the parvocellular pathway [35,37], and approximately $80 \%$ of ganglion cells are parvocellular cells [40].

The PERG is a mass potential that sums information primarily from the electrical potential of retinal ganglion cells $[41,42]$. It was suggested that the PERG response measured retinal ganglion cell activity; therefore, the direct loss of ganglion cell counts may affect the PERG response. In the present study, we also found a significant PERG amplitude reduction in NDR patients, which correlated with decreased superior macular GCC thickness. These results support the theory that the PERG relied on a centrally presented stimulus, in particular GCC thickness, and was strongly associated with macular parameters. However, the significant association between PERG amplitude and peripapillary inferior RNFL thickness in the NDR group also suggested that the PERG was measuring more than a central response [43]. For PERG implicit times, we did not observe any significant change or correlation with retinal inner layer thickness. A previous study suggested that a delay in ERG implicit times correlated with the chance of onset and development of DR [44]. Thus, there was a slight increase in implicit times in the NDR group, which was not significant, because in this study we only evaluated diabetic patients without DR.

There are several limitations of the present study. First, the sample size was small, although significant GCC changes were measured in NDR patients. Second, most of the diabetic patients we included in this study had controlled blood glucose levels, and some were receiving insulin therapy. It has been suggested that insulin can reverse retinal apoptosis and provide trophic support for retinal neurons [45]. The changes in GCC and RNFL thicknesses between NDR patients and control subjects may be larger because untreated diabetics were not included. Furthermore, the SD OCT could not provide a sectorial analysis of macular GCC; therefore we evaluated only the more limited data of GCC (average, superior, and inferior GCC thickness). If subdivided macular GCC data were available, the results could be more conclusive.

In summary, our results provided proof of concept that early neuronal degeneration existed in the inner retina in diabetic patients before the onset of clinical DR. SD OCT demonstrated that macular GCC thickness decreased much earlier than peripapillary RNFL thinning. In addition, correlations between GCC thicknesses and contrast sensitivities in NDR patients suggested that a loss of parvocellular cells may be involved in macular GCC damage. However, whether the macular GCC changes in diabetic eyes were a result of the effect of vascular diabetic retinopathy or whether they were primarily caused by direct neurological damage from chronic hyperglycemia requires further study.

This work was supported by the National Natural Science Foundation of China (81341029).

1 UK Prospective Diabetes Study (UKPDS) Group. Effect of intensive blood-glucose control with metformin on complications in overweight patients with type 2 diabetes (UKPDS 34). Lancet, 1988, 352: 854-865

2 Barber AJ, Lieth E, Khin SA, Antonetti DA, Buchanan AG, Gardner TW. Neural apoptosis in the retina during experimental and human diabetes. Early onset and effect of insulin. J Clin Invest, 1998, 102: 783-791

3 Takano M, Sango K, Horie H, Sato M, Iijima Y, Ohno S, Ihno S, Ishikawa Y. Diabetes alters neurite regeneration from mouse retinal explants in culture. Neurosci Lett, 1999, 275: 175-178

4 Asnaghi V, Gerhardinger C, Hoehn T, Adeboje A, Lorenzi M. A role for the polyol pathway in the early neuroretinal apoptosis and glial changes induced by diabetes in the rat. Diabetes, 2003, 52: 506-511

5 Martin PM, Roon P, Van Ells TK, Ganapathy V, Smith SB. Death of retinal neurons in streptozotocin-induced diabetic mice. Invest Ophthalmol Vis Sci, 2004, 45: 3330-3336

6 Fletcher EL, Phipps JA, Wilkinson-berka JL. Dysfunction of retinal neurons and glia during diabetes. Clin Exp Optom, 2005, 3: 132-145

7 Goebel W, Kretzchmar-Gross T. Retinal thickness in diabetic retinopathy: a study using optical coherence tomography (OCT). Retina, 2002, 22: 759-767 
8 Sugimoto M, Sasoh m, Ido M, Wakitani Y, Takahashi C, Uji Y. Detection of early diabetic change with optical coherence tomography in type 2 diabetes mellitus patients without retinopathy. Ophthalmologica, 2005, 219: 379-385

9 Lopes de Faria JM, Russ H, Costa VP. Retinal nerve fiber layer loss in patients with type 1 diabetes mellitus without retinopathy. $\mathrm{Br} \mathrm{J}$ Ophthalmol, 2002, 86: 725-728

10 Tyberg M, Lindblad U, Melander A, Lovestam-Adrian M, Ponjavic $\mathrm{V}$, Andréasson S. Electrophysiological studies in newly onset type 2 diabetes without visible vascular retinopathy. Doc Ophthalmol, 2011, 123: $193-198$

11 Bronson-Castain KW, Bearse MA Jr, Neuville J, Jonasdottir S, King-Hooper B, Barez S, Schneck ME, Adams AJ. Adolescents with Type 2 diabetes: early indications of focal retinal neuropathy, retinal thinning, and venular dilation. Retina, 2009, 29: 618-626

12 Di Leo MA, Caputo S, Falsini B, Porciatti V, Minnella A, Greco AV, Ghirlanda G. Nonselective loss of contrast sensitivity in visual system testing in early type I diabetes. Diabetes Care, 1992, 15: 620-625

13 Várkonyi TT, Petõ T, Dégi R, Keresztes K, Lengyel C, Janáky M, Kempler P, Lonovics J. Impairment of visual evoked potentials: an early central manifestation of diabetic neuropathy? Diabetes Care, 2002, 25: 1661-1662

14 Abraham FA, Haimovitz J, Berezin M. The photopic and scotopic visual thresholds in diabetics without diabetic retinopathy. Metab Pediatr Syst Ophthalmol, 1988, 11: 76-77

15 Wilkinson CP, Ferris FL 3rd, Klein RE, Lee PP, Agardh CD, Davis M, Dills D, Kampik A, Pararajasegaram R, Verdaguer JT; Global Diabetic Retinopathy Project Group. Proposed international clinical diabetic retinopathy and diabetic macular edema disease severity scales. Ophthalmology, 2003, 110: 1677-1682

16 Bach M1, Brigell MG, Hawlina M, Holder GE, Johnson MA, McCulloch DL, Meigen T, Viswanathan S. ISCEV standard for clinical pattern electroretinography. Doc Ophthalmol, 2007, 114: $111-126$

17 Antonetti DA, Barber AJ, Bronson SK, Freeman WM, Gardner TW, Jefferson LS, Kester M, Kimball SR, Krady JK, LaNoue KF, Norbury CC, Quinn PG, Sandirasegarane L, Simpson IA; JDRF Diabetic Retinopathy Center Group. Diabetic retinopathy: seeing beyond glucose-induced microvascular disease. Diabetes, 2006, 55: 2401-2411

18 Kim NR, Kim JH, Lee J, Lee ES, Seong GJ, Kim CY. Determinants of perimacular inner retinal layer thickness in normal eyes measured by fourier-domain optical coherence tomography. Invest Ophthalmol Vis Sci, 2011, 52: 3414-3418

19 Demirkaya N, van Dijk HW, van Schuppen SM, Abràmoff MD, Garvin MK, Sonka M, Schlingemann RO, Verbraak FD. Effect of age on individual retinal layer thickness in normal eyes as measured with spectral-domain optical coherence tomography. Invest Ophthalmol Vis Sci, 2013, 54: 4934-4940

20 Stela Vujosevic, Midena Edoardo. Retinal layers changes in human preclinical and early clinical diabetic retinopathy support early retinal neuronal and Müller cells alterations. J Diabetes Res, 2013, 2013: 905058

21 Oshitari T, Hanawa K, Adachi-Usami E. Changes of macular and RNFL thicknesses measured by Stratus OCT in patients with early stage diabetes. Eye (Lond), 2009, 23: 884-889

22 Park HY, Kim IT, Park CK. Early diabetic changes in the nerve fibre layer at the macula detected by spectral domain optical coherence tomography. Br J Ophthalmol, 2011, 95: 1223-1228

23 Peng PH, Lin HS, Lin S. Nerve fibre layer thinning in patients with preclinical retinopathy. Can J Ophthalmol, 2009, 44: 417-422

24 Verma A, Raman R, Vaitheeswaran K, Pal SS, Laxmi G, Gupta M, Shekar SC, Sharma T. Does neuronal damage precede vascular damage in subjects with Type 2 diabetes mellitus and having no clinical diabetic retinopathy? Ophthalmic Res, 2012, 47: 202-207

25 Blumenthal EZ, Williams JM, Weinreb RN, Girkin CA, Berry CC, Zangwill LM. Reproducibility of nerve fiber layer thickness measurements by use of optical coherence tomography. Ophthalmology,
2000, 107: 2278-2282

26 Paunescu LA, Schuman JS, Price LL, Stark PC, Beaton S, Ishikawa H, Wollstein G, Fujimoto JG. Reproducibility of nerve fiber thickness, macular thickness, and optical nerve head measurements using StratusOCT. Invest Ophthalmol Vis Sci, 2004, 45: 1716-1724

27 Savini G, Zanini M, Carelli V, Sadun AA, Ross-Cisneros FN, Barboni $\mathrm{P}$. Correlation between retinal nerve fibre layer thickness and optic nerve head size: an optical coherence tomography study. Br J Ophthalmol, 2005, 89: 489-492

28 Hood DC, Fortune B, Arthur SN, Xing D, Salant JA, Ritch R, Liebmann JM. Blood vessel contributions to retinal nerve fiber layer thickness profiles measured with optical coherence tomography. J Glaucoma, 2008, 17: 519-528

29 Kim NR, Lee ES, Seong GJ, Kim JH, An HG, Kim CY. Structure-function relationship and diagnostic value of macular ganglion cell complex measurement using Fourier-domain OCT in glaucoma. Invest Ophthalmol Vis Sci, 2010, 51: 4646-4651

30 van Dijk HW, Verbraak FD, Kok PH, Garvin MK, Sonka M, Lee K, Devries JH, Michels RP, van Velthoven ME, Schlingemann RO, Abràmoff MD. Decreased retinal ganglion cell layer thickness in patients with type 1 diabetes. Invest Ophthalmol Vis Sci, 2010, 51: 3660-3665

31 van Dijk HW, Verbraak FD, Kok PH, Stehouwer M, Garvin MK, Sonka M, DeVries JH, Schlingemann RO, Abràmoff MD. Early neurodegeneration in the retina of type 2 diabetic patients. Invest Ophthalmol Vis Sci, 2012, 53: 2715-2719

32 Quigley HA, Addicks EM. Quantitative studies of retinal nerve fiber layer defects. Arch Ophthalmol, 1982, 100: 807-814

33 Harwerth RS, Wheat JL, Fredette MJ, Anderson DR. Linking structure and function in glaucoma. Prog Retin Eye Res, 2010, 29: 249-271

34 Kern TS, Engerman RL. Vascular lesions in diabetes are distributed non-uniformly within the retina. Exp Eye Res, 1995, 60: 545-549

35 Ewing FM, Deary IJ, Strachan MW, Frier BM. Seeing beyond retinopathy in diabetes: electrophysiological and psychophysical abnormalities and alterations in vision. Endocr Rev, 1998, 19: 462-476

36 North RV, Farrell U, Banford D, Jones C, Gregory JW, Butler G, Owens DR. Visual function in young IDDM patients over 8 years of age: a 4-year longitudinal study. Diabetes Care, 1997, 20: 1724-1730

37 Fletcher EL, Phipps JA, Ward MM, Puthussery T, Wilkinson-Berka JL. Neuronal and glial cell abnormality as predictors of progression of diabetic retinopathy. Curr Pharmaceut Design, 2007, 13: 2699-2712

38 Dosso AA, Yenice-Ustun F, Sommerhalder J, Golay A, Morel Y, Leuenberer PM. Contrast sensitivity in obese dyslipidemic patients with insulin resistance. Arch Ophthalmol, 1998, 116: 1316-1320

39 Lieth E, Gardner TW, Barber AJ, Antonetti DA; Penn State Retina Research Group. Retinal neurodegeneration: early pathology in diabetes. Clin Exp Ophthalmol, 2000, 26: 3-8

40 Shapley R, Perry VH. Cat and monkey retinal ganglion cells and their visual functional roles. Trends Neurosci, 1986, 9: 229-235

41 Maffei L, Fiorentini A. Electroretinographic responses to alternating gratings before and after section of the optic nerve. Science, 1981, 211: 953-955

42 Hood DC, Frishman LJ, Viswanathan S, Robson JG, Ahmed J. Evidence for a ganglion cell contribution to the primate electroretinogram (ERG): effects of TTX on the multifocal ERG in macaque. Vis Neurosci, 1999, 16: 411-416

43 Ventura LM, Porciatti V. Restoration of retinal ganglion cell function in early glaucoma after intraocular pressure reduction: a pilot study. Ophthalmology, 2005, 112: 20-27

44 Ng JS, Bearse MA Jr, Schneck ME, Barez S, Adams AJ. Local diabetic retinopathy prediction by multifocal ERG delays over 3 years. Invest Ophthalmol Vis Sci, 2008, 49: 1622-1628

45 Reiter CE, Gardner TW. Functions of insulin and insulin receptor signaling in retina: possible implications for diabetic retinopathy. Prog Retin Eye Res, 2003, 22: 545-562

Open Access This article is distributed under the terms of the Creative Commons Attribution License which permits any use, distribution, and reproduction in any medium, provided the original author(s) and source are credited. 